\title{
Violência contra a mulher em uma cidade da fronteira do Brasil
}

\author{
Violence against women in a city on the frontier of Brazil \\ Violencia contra las mujeres en una ciudad de la frontera de Brasil
}

Recebido: 13/02/2021 | Revisado: 20/02/2021 | Aceito: 23/02/2021 | Publicado: 02/03/2021

\author{
Ana Caroline Garcia Sebaldeli \\ ORCID: https://orcid.org/ 0000-0002-3737-9670 \\ Universidade do Estado de Mato Grosso, Brasil \\ E-mail: carol.sebaldeli@hotmail.com \\ Eliane Ignotti \\ ORCID: https://orcid.org/0000-0002-9743-1856 \\ Universidade do Estado de Mato Grosso, Brasil \\ E-mail: eliane.ignotti@unemat.br \\ Shaiana Vilella Hartwig \\ ORCID: https://orcid.org/0000-0003-4245-2163 \\ Universidade do Estado de Mato Grosso, Brasil \\ E-mail: shaiana.hartwig@unemat.br
}

\begin{abstract}
Resumo
A violência contra as mulheres é um problema de saúde pública. Objetiva-se descrever a incidência e o perfil das mulheres vítimas de violência física e sexual na cidade de Cáceres - MT. Estudo descritivo, do tipo transversal, sobre os casos de violência física e sexual registrados contra a mulher em Cáceres-MT de janeiro a dezembro de 2018. Como fonte de dados utilizou-se dos registros dos boletins de ocorrências da Delegacia Especial de Atendimento à Mulher (DEAM) e da Secretaria de Vigilância em Saúde por meio do Sistema de Informação de Agravos de Notificação (SINAN). Observou-se 296 registros de mulheres que sofreram algum tipo de violência física ou sexual, dos quais 274 constavam apenas na DEAM e 15 apenas no SINAN. De acordo com a DEAM o tipo de violência mais frequente foi lesão corporal $(59,4 \%)$ e segundo o SINAN, o tipo de violência mais sofrido pelas mulheres foi violência física $(54,5 \%)$. Com relação ao perfil a maioria das mulheres que sofreram violência são pardas, com idade entre 21 a 30 anos, casadas ou conviventes, com ensino fundamental e do lar. A violência física foi o tipo de violência mais frequente seguida de violência sexual. Este estudo aponta discordância entre os registros de violência contra a mulher, sugerindo importante subnotificação do agravo, além de falta de integração das áreas de segurança pública e de saúde.
\end{abstract}

Palavras-chave: Violência contra a mulher; Notificação; Vigilância em saúde pública.

\begin{abstract}
Violence against women is a public health problem. The objective is to describe the incidence and profile of women victims of physical and sexual violence in the city of Cáceres - MT. Descriptive, cross-sectional study on cases of physical and sexual violence registered against women in Cáceres-MT from January to December 2018. As a data source, we used the records of occurrences from the Special Police Station for Women. (DEAM) and the Health Surveillance Secretariat through the Notifiable Diseases Information System (SINAN). There were 296 records of women who suffered some type of physical or sexual violence, of which 274 were recorded only in DEAM and 15 only in SINAN. According to DEAM, the most frequent type of violence was bodily injury (59.4\%) and according to SINAN, the type of violence most suffered by women was physical violence (54.5\%). Regarding the profile, the majority of women who suffered violence are brown, aged between 21 and 30 years, married or living with elementary and home education. Physical violence was the most frequent type of violence followed by sexual violence. This study points out disagreement between the records of violence against women, suggesting an important underreporting of the condition, in addition to the lack of integration in the areas of public security and health.
\end{abstract}

Keywords: Violence against women; Notification; Public health surveillance.

\section{Resumen}

La violencia contra la mujer es un problema de salud pública. El objetivo es describir la incidencia y perfil de las mujeres víctimas de violencia física y sexual en la ciudad de Cáceres - MT. Estudio descriptivo, transversal, de los casos de violencia física y sexual registrados contra mujeres en Cáceres-MT de enero a diciembre de 2018. Como fuente de datos se utilizaron los registros de ocurrencias de la Comisaría Especializada de la 
Mujer (DEAM) y la Secretaría de Vigilancia Sanitaria a través del Sistema de Información de Enfermedades Notificables (SINAN). Hubo 296 registros de mujeres que sufrieron algún tipo de violencia física o sexual, de las cuales 274 fueron registradas solo en DEAM y 15 solo en SINAN. Según la DEAM, el tipo de violencia más frecuente fueron las lesiones corporales $(59,4 \%)$ y según el SINAN, el tipo de violencia más sufrida por las mujeres fue la violencia física $(54,5 \%)$. En cuanto al perfil, la mayoría de las mujeres que sufrieron violencia son morenas, con edades comprendidas entre los 21 y 30 años, casadas o que viven con educación primaria y domiciliaria. La violencia física fue el tipo de violencia más frecuente seguida de la violencia sexual. Este estudio señala desacuerdo entre los registros de violencia contra las mujeres, sugiriendo un importante subregistro de la condición, además de la falta de integración en las áreas de seguridad pública y salud.

Palabras clave: Violencia contra la mujer; Notificación; Vigilancia de la salud pública.

\section{Introdução}

Violência é um agravo definido como uso intencional da força ou do poder que resulte ou possa resultar em lesão, morte, dano psicológico, deficiência de desenvolvimento e privação (WHO, 1996).

A violência contra as mulheres também conhecida como violência de gênero ou violência doméstica, é um problema de saúde pública pela magnitude de sua prevalência, gravidade e recorrência, assim como pelas consequências negativas na saúde física, mental, sexual e reprodutiva das mulheres (Vieira, et al., 2013; Semahegn \& Mengistie, 2015). Atinge mulheres de todo o mundo e possui diversas causas, dentre elas as sociais, políticas, econômicas e os fatores biológicos. Este tipo de violência geralmente ocorre na residência e tem como principal agressor o companheiro (Silva \& Oliveira, 2015).

Com a criação da Lei 11.340/2006, conhecida como Maria da Penha, a violência contra a mulher passou a ter maior destaque. Essa lei classifica a violência como física, sexual, psicológica, moral ou patrimonial. A lei cria mecanismos para coibir e prevenir a violência contra a mulher, independente de classe, raça, etnia ou orientação sexual (Brasil, 2006). Desta forma assegura a toda mulher viver sem violência, preservar a saúde física, mental, moral, intelectual e social, assim como condições para o exercício efetivo dos direitos a vida, à segurança e à saúde (Meneghel, et al., 2013; Silva \& Oliveira, 2015).

A violência contra a mulher tem como principal agressor o parceiro íntimo, sendo o risco de violência dentro do seu lar maior que o risco de ser vítima de violência na rua. Cerca de 10 a $50 \%$ das mulheres em todo o mundo já sofreram alguma forma de violência física, praticada por seus companheiros (Marinheiro, et al., 2006).

O mapa da violência mostra que o Brasil ocupou a quinta posição entre os países com uma taxa de homicídios por 100 mil mulheres no ano de 2013 e o estado de Mato Grosso apresentou uma taxa de 5,8 homicídio por 100 mil mulheres neste mesmo ano, estando entre os estados mais violentos (Waiselfisz, 2015). A pesquisa realizada pelo Fórum Brasileiro de Segurança Pública revelou que 29\% das mulheres sofreram violência ou agressão e $40 \%$ sofreram assédio, sendo 503 agressões por hora (Brasil, 2019).

A violência física representa $60 \%$ dos atendimentos e tem uma maior incidência nas mulheres jovens e adultas (Waiselfisz, 2015). Definida como o uso da força física ou de algum tipo de arma podendo provocar lesões contra si mesmo ou outra pessoa (Brasil, 2002). 
A violência sexual representa 11,9\% dos atendimentos estando em terceiro lugar quando comparada aos outros tipos de violência. Há maior incidência desse tipo de violência ocorre nas crianças com menos de 11 anos de idade e nos adolescentes (Waiselfisz, 2015). Segundo o Ministério da Saúde violência sexual é quando uma pessoa obriga a outra ao ato sexual sem a sua vontade, por meio de força física e do poder ou que a exponha em interações sexuais da qual o agressor tenta obter gratificação (Brasil, 2002).

Uma das dificuldades da inclusão da violência nos serviços de saúde se associa a invisibilidade do fenômeno pelos profissionais de saúde, uma vez que se o problema não existe ele não se coloca como problemática geradora de práticas de saúde. A notificação é um importante instrumento de política pública, uma vez que dimensiona a questão da violência e permite o conhecimento da violência doméstica. A conscientização da sua importância, a quebra de ideias pré-concebidas e o treinamento correto para diagnosticar situações de violência são necessárias para que o profissional de saúde seja capaz de detectar e notificar essa realidade que se apresenta de forma tão expressiva no cotidiano (Saliba, et al., 2007; Souza, et al., 2018).

A violência contra a mulher é um tema de interesse mundial, pois consiste em um problema de saúde pública, repercutindo em toda a sociedade. O Brasil é um país que apresenta taxas crescente de violência. Essas altas taxas podem estar relacionadas com os fatores socioeconômicos, baixa escolaridade, infraestrutura precária e o desemprego.

O serviço de saúde é um setor privilegiado por entrar em contato com a mulher em algum momento da sua vida, por isso os profissionais de saúde são uma ferramenta importante para reconhecer as vítimas de violência e dessa forma poder ajudá-las e prevenir que outros problemas de saúde venham a ocorrer devido à violência sofrida.

Justifica-se o presente estudo para dar maior visibilidade ao problema no município de Cáceres-MT e dessa forma reduzir a negligência dos profissionais de saúde em relação a violência contra a mulher e chamar atenção para a importância da notificação. Nesse contexto, o presente estudo busca analisar os registros de violência contra a mulher, bem como o perfil das vítimas na cidade de Cáceres - MT.

\section{Metodologia}

Estudo epidemiológico descritivo, do tipo transversal, quantitativo, sobre os casos de violência contra a mulher registrados nos serviços de saúde e de segurança pública. As pesquisas epidemiológicas descritivas têm como foco descrever os dados para caracterizar uma população (Gil, 2017). O método quantitativo, faz-se a coleta de dados quantitativos ou numéricos, para serem analisados por meio de técnicas de porcentagens, estatísticas e probabilidades (Pereira, et al., 2018).

O estudo foi desenvolvido no município de Cáceres-MT, cidade localizada à $225 \mathrm{~km}$ da capital Cuiabá-MT, compondo a região sudoeste do estado de Mato Grosso, com aproximadamente 93.882 habitantes, sendo aproximadamente 46.000 mulheres (49\%) (IBGE, 2018).

Foram utilizados dados extraídos dos boletins de ocorrências da Delegacia Especial de Atendimento à Mulher (DEAM) e dados da Secretaria de Vigilância em Saúde a partir de registros do Sistema de Informação de Agravos de Notificação (SINAN), através da ficha de notificação/investigação pessoal de violência doméstica, sexual e/ou outras violências interpessoais, registrados no período de janeiro de 2018 a dezembro de 2018.

Foram escolhidos dois bancos de dados a fim de trabalhar com os dados mais próximos da incidência no mundo real, porém alguns registros podem ser perdidos, pois nem todas as vítimas de violência que registram o boletim de ocorrência procuram atendimento médico, bem como nem todas as vítimas que procuram atendimento médico realizam o boletim de ocorrência.

Os dados foram coletados através dos Boletins de Ocorrência registrados na DEAM e através da ficha de notificação de Violência Interpessoal/Autoprovocada do SINAN. 
Como variáveis de estudos foram consideradas sobre a vítima e sobre o agressor: idade, cor da pele/raça, escolaridade, profissão e estado civil.

Para o banco da DEAM foram registrados em 2018, 539 boletins de ocorrência de violência contra a mulher, os tipos de violência registrados foram: ameaça, injúria, lesão corporal, estupro, tentativa de homicídio e outros. Optou-se por selecionar as violências classificadas como violência física, para essa classificação foram selecionados todas os tipos de violência registrados com o termo lesão corporal podendo ser associado a outro tipo de violência; e para violência sexual foram selecionados os tipos de violência com o termo estupro. A seleção para esses tipos de violência, se deve ao fato de serem os mais citados na literatura como os mais prevalentes, e por possivelmente serem as violências que procurariam os serviços de saúde.

Para o banco do SINAN foram inclusos todos os tipos de violência registrados: violência física, psicológica/moral e sexual, excluindo-se as violências autoprovocadas.

Foi elaborado um banco de dados constituído numa planilha eletrônica no Microsoft Excel 2017. Os dados foram analisados por meio de estatística descritiva, frequências absolutas e relativas. Para o cálculo da incidência foi utilizada a seguinte fórmula:

\section{Incidência $=\frac{\text { Número de casos novos do tipo de violência }}{\text { Número total de casos de violência }} \times 100$}

Dos casos selecionados não foram encontrados casos recorrentes (duplicados) de violência contra a mulher, por isso o cálculo de incidência foi o escolhido, por todos os casos serem considerados novos daquele ano.

O processamento, análise dos dados e construção de figuras aconteceu através programa Microsoft Excel 2017. Para checagem da concordância entre os bancos foram feitas em três verificações em todos os registros, independentemente do tipo de agressões sofrida para que não houvesse dados perdidos devido a seleção do tipo de agressão.

O estudo desenvolvido foi pautado nas recomendações da Resolução do Conselho Nacional de Ética em Pesquisa com Seres Humanos n ${ }^{\circ}$ 466/12. Sendo realizado somente após aprovação pelo Comitê de Ética em Pesquisa da Universidade do estado de Mato Grosso sob o parecer n ${ }^{\circ}$ 3.501.929, autorização da Delegada responsável pela DEAM e do secretário de saúde do município, mediante assinatura do Termo de Consentimento Livre Esclarecido.

\section{Resultados}

O número de mulheres que sofreram violência do tipo lesão corporal e estupro no município de Cáceres, em 2018, segundo a Delegacia Especializada no Atendimento à Mulher (DEAM) foram 281 e de acordo com o Sistema de Informação de Agravos de Notificação (SINAN) foram 22 casos de violência.

O tipo de violência contra as mulheres é classificado de maneira diferente pela DEAM e pelo SINAN. Segundo a DEAM, o tipo de violência mais sofrido pelas mulheres na cidade de Cáceres, no ano de 2018, foi lesão corporal (59,4\%), seguido de lesão corporal e outras violências $(23,8 \%)$ e estupro de vulnerável $(13,9 \%)$. Segundo o SINAN, o tipo de violência mais sofrido pelas mulheres foi violência física (54,5\%), seguido de física e psicológica (27,3\%), física e sexual $(9,1 \%)$ e psicológica e sexual $(9,1 \%)$ (Figura 1). 
Figura 1. Incidência do tipo de violência contra a mulher segundo local de coleta dos dados (A) Delegacia Especializada no Atendimento à Mulher e (B) Sistema de Informação de Agravos de Notificação em Cáceres-MT, 2018.

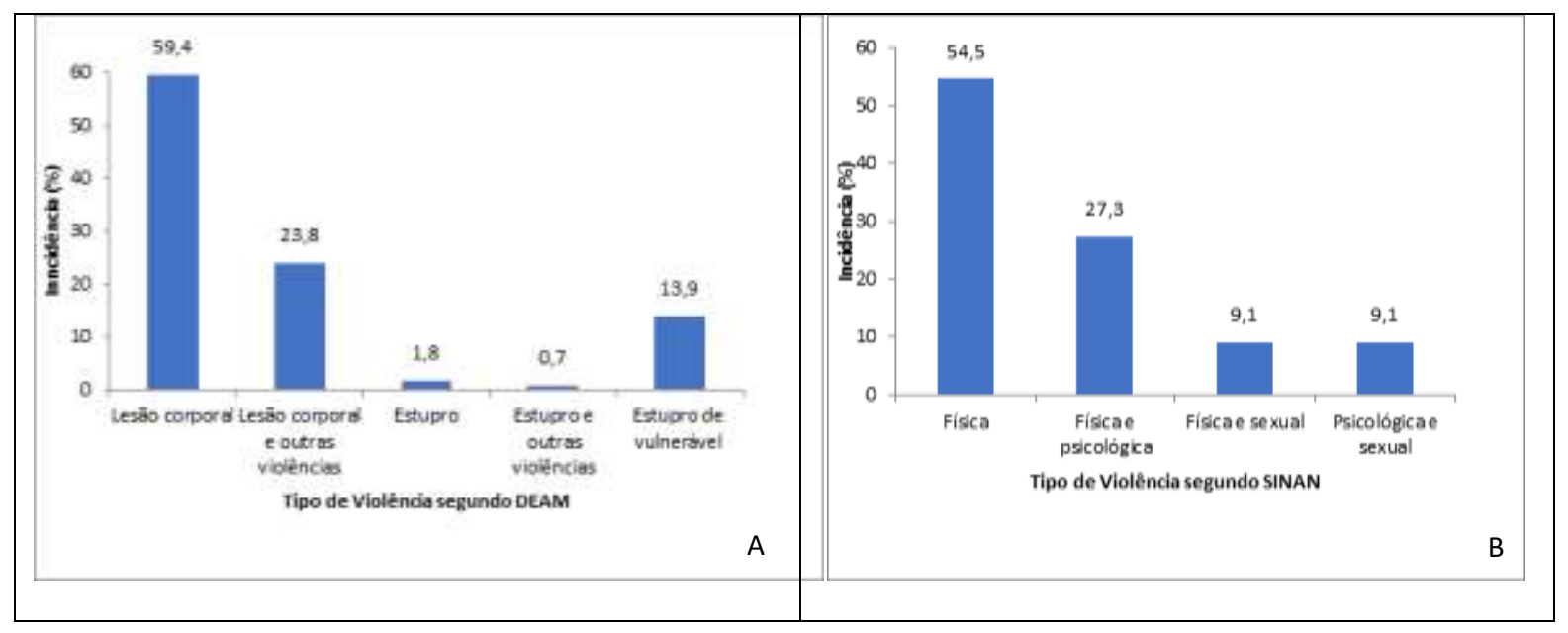

Fonte: DEAM (2018) e SINAN (2018).

O perfil das mulheres que sofrem violência no município de Cáceres é semelhante entre os bancos de dados da DEAM e do SINAN sendo a maioria parda, com idade entre 21 a 30 anos, casadas ou conviventes, com ensino fundamental e do lar.

É importante ressaltar que as variáveis a data de nascimento são de preenchimento obrigatório e, portanto, têm-se nelas a exata representatividade no que se propõe. Quando se consideram outras informações, como raça/cor da pele, escolaridade, situação conjugal, profissão, torna-se evidente um elevado número de campos em branco nos dados da DEAM. Quanto a incompletude dos dados, 53\% escolaridade; 54\% dos campos relativos a profissão; 34\% quanto a raça/cor; e, 12\% relacionado a situação conjugal (Tabela 1). 
Tabela 1. Perfil das vítimas de violência contra a mulher segundo local de coleta dos dados no município de Cáceres-MT, 2018 .

\begin{tabular}{|c|c|c|c|c|}
\hline \multirow{2}{*}{\begin{tabular}{|l|} 
Variáveis \\
Faixa etária
\end{tabular}} & \multicolumn{2}{|c|}{ Dados DEAM } & \multicolumn{2}{|c|}{ Dados SINAN } \\
\hline & $\mathbf{N}$ & $\%$ & $\mathbf{N}$ & $\%$ \\
\hline Média \pm Desvio Padrão & $28,6 \pm 13,5$ & & $31 \pm 13$ & \\
\hline$<10$ & 13 & 4,6 & 1 & 4,5 \\
\hline $11-20$ & 67 & 23,8 & 4 & 18,1 \\
\hline $21-30$ & 82 & 29,1 & 7 & 31,8 \\
\hline $31-40$ & 61 & 21,7 & 5 & 22,7 \\
\hline $41-50$ & 29 & 10,3 & 2 & 9,0 \\
\hline$>50$ & 15 & 5,3 & 3 & 13,6 \\
\hline Não informou & 14 & 4,9 & - & - \\
\hline \multicolumn{5}{|l|}{ Raça } \\
\hline Branca & 28 & 9,9 & 2 & 9,0 \\
\hline Preta & 8 & 2,8 & - & - \\
\hline Parda & 147 & 52,3 & 19 & 86,3 \\
\hline Indígena & - & - & 1 & 4,5 \\
\hline Não informou & 98 & 34,8 & - & - \\
\hline \multicolumn{5}{|l|}{ Estado civil } \\
\hline Solteira & 107 & 38,0 & 9 & 40,9 \\
\hline Casada/Convivente & 119 & 42,3 & 9 & 40,9 \\
\hline Viúva & 7 & 2,4 & 1 & 4,5 \\
\hline Divorciada/Separada & 13 & 4,6 & 2 & 9,0 \\
\hline Não informou & 35 & 12,4 & 1 & 4,5 \\
\hline \multicolumn{5}{|l|}{ Escolaridade } \\
\hline Não alfabetizada & 6 & 2,1 & - & - \\
\hline Alfabetizada & 32 & 11,3 & - & - \\
\hline Ensino fundamental & 38 & 13,5 & 10 & 45,4 \\
\hline Ensino médio & 50 & 17,7 & 9 & 40,9 \\
\hline Ensino superior & 6 & 2,1 & - & \\
\hline Não informou & 149 & $\mathbf{5 3 , 0}$ & 3 & 13,6 \\
\hline Profissão & & & Dadc & nivel \\
\hline $\begin{array}{l}\text { Membros superiores do poder } \\
\text { público, dirigentes de } \\
\text { organizações de interesse } \\
\text { público e de empresas, } \\
\text { gerentes }\end{array}$ & 2 & 0,7 & & \\
\hline $\begin{array}{l}\text { Profissionais das ciências e das } \\
\text { artes }\end{array}$ & 2 & 0,7 & & \\
\hline Técnicos de nível médio & 4 & 1,4 & & \\
\hline $\begin{array}{l}\text { Trabalhadores de serviços } \\
\text { administrativos }\end{array}$ & 2 & 0,7 & & \\
\hline $\begin{array}{l}\text { Trabalhadores dos serviços, } \\
\text { vendedores do comércio em } \\
\text { lojas e mercados }\end{array}$ & 32 & 11,3 & & \\
\hline $\begin{array}{l}\text { Trabalhadores da produção de } \\
\text { bens e serviços industriais }\end{array}$ & 1 & 0,3 & & \\
\hline Aposentada & 4 & 1,4 & & \\
\hline Estudante & 30 & 10,6 & & \\
\hline Do lar & 47 & 16,7 & & \\
\hline Autônomo & 3 & 1,0 & & \\
\hline Desempregado & 2 & 0,7 & & \\
\hline Não informou & 152 & 54,0 & & \\
\hline TOTAL & 281 & 99,9 & 22 & 99,9 \\
\hline
\end{tabular}

Fonte: DEAM (2018) e SINAN (2018).

Em relação ao perfil dos agressores contra as mulheres, segundo o banco de dados da DEAM, no município de Cáceres - MT são homens (100\%), de raça/cor da pele parda (43,4\%), com idade entre 31 a 40 anos (28,8\%), 
casados/conviventes $(44,1 \%)$, com ensino fundamental (14,5\%) e trabalhadores de serviços, vendedores do comércio em lojas e mercados (14,9\%). Observa-se grande número de dados não informados como escolaridade (64\%), profissão (59,7\%), cor/raça $(43 \%)$ e estado civil (25\%). (Tabela 2$)$.

Tabela 2. Perfil dos agressores de violência contra a mulher no município de Cáceres-MT, 2018.

\begin{tabular}{|c|c|c|}
\hline \multirow{2}{*}{$\begin{array}{l}\text { Variáveis } \\
\text { Faixa etária }\end{array}$} & \multicolumn{2}{|c|}{ Dados DEAM } \\
\hline & $\mathbf{N}$ & $\%$ \\
\hline Média \pm Desvio Padrão & $34,7 \pm 10.9$ & \\
\hline $10-20$ & 16 & 5,6 \\
\hline $21-30$ & 69 & 24,5 \\
\hline $31-40$ & 81 & 28,8 \\
\hline $41-50$ & 38 & 13,5 \\
\hline $51-60$ & 16 & 5,6 \\
\hline$>60$ & 6 & 2,1 \\
\hline Não informou & 55 & 19,5 \\
\hline \multicolumn{3}{|l|}{ Raça } \\
\hline Branca & 22 & 7,8 \\
\hline Preta & 16 & 5,6 \\
\hline Parda & 122 & 43,4 \\
\hline Indígena & - & - \\
\hline Não informou & 121 & 43,0 \\
\hline \multicolumn{3}{|l|}{ Estado civil } \\
\hline Solteiro & 66 & 23,4 \\
\hline Casado/convivente & 124 & 44,1 \\
\hline Viúvo & - & - \\
\hline Divorciado/Separado & 18 & 6,4 \\
\hline Não informou & 73 & 25,9 \\
\hline \multicolumn{3}{|l|}{ Escolaridade } \\
\hline Não alfabetizada & 3 & 1,0 \\
\hline Alfabetizada & 19 & 6,7 \\
\hline Ensino fundamental & 41 & 14,5 \\
\hline Ensino médio & 34 & 12,0 \\
\hline Ensino superior & 4 & 1,4 \\
\hline Não informou & 180 & 64,0 \\
\hline \multicolumn{3}{|l|}{ Profissão } \\
\hline $\begin{array}{l}\text { Membros das forças armadas, } \\
\text { policiais e bombeiros militares }\end{array}$ & 2 & 0,7 \\
\hline $\begin{array}{l}\text { Membros superiores do poder } \\
\text { público, dirigentes de organizações } \\
\text { de interesse público e de empresas, } \\
\text { gerentes }\end{array}$ & 1 & 0,3 \\
\hline $\begin{array}{l}\text { Profissionais das ciências e das } \\
\text { artes }\end{array}$ & 2 & 0,7 \\
\hline Técnicos de nível médio & 1 & 0,3 \\
\hline $\begin{array}{l}\text { Trabalhadores dos serviços, } \\
\text { vendedores do comércio em lojas } \\
\text { e mercados }\end{array}$ & 42 & 14,9 \\
\hline $\begin{array}{l}\text { Trabalhadores agropecuários, } \\
\text { florestais e da pesca }\end{array}$ & 7 & 2,4 \\
\hline $\begin{array}{l}\text { Trabalhadores da produção de } \\
\text { bens e serviços industriais }\end{array}$ & 21 & 7,4 \\
\hline $\begin{array}{l}\text { Trabalhadores em serviços de } \\
\text { reparação e manutenção }\end{array}$ & 10 & 3,3 \\
\hline Aposentada & 8 & 2,8 \\
\hline Estudante & 3 & 1,0 \\
\hline Autônomo & 8 & 2,8 \\
\hline Desempregado & 10 & 3,5 \\
\hline Não informou & 168 & $\mathbf{5 9 , 7}$ \\
\hline TOTAL & 281 & 99,9 \\
\hline
\end{tabular}

Fonte: DEAM (2018). 
Segundo a DEAM, os meses que ocorreram mais casos de violência contra a mulher foram março (35), maio (31) e junho (31). Segundo o SINAN, o mês que houve predominância de casos foi dezembro (7), único mês com mais casos notificados no SINAN do que da DEAM. No mês de maio e julho o SINAN não registrou nenhuma notificação (Figura 2).

Figura 2. Distribuição mensal da ocorrência da violência contra a mulher segundo local de notificação, Cáceres-MT, 2018.

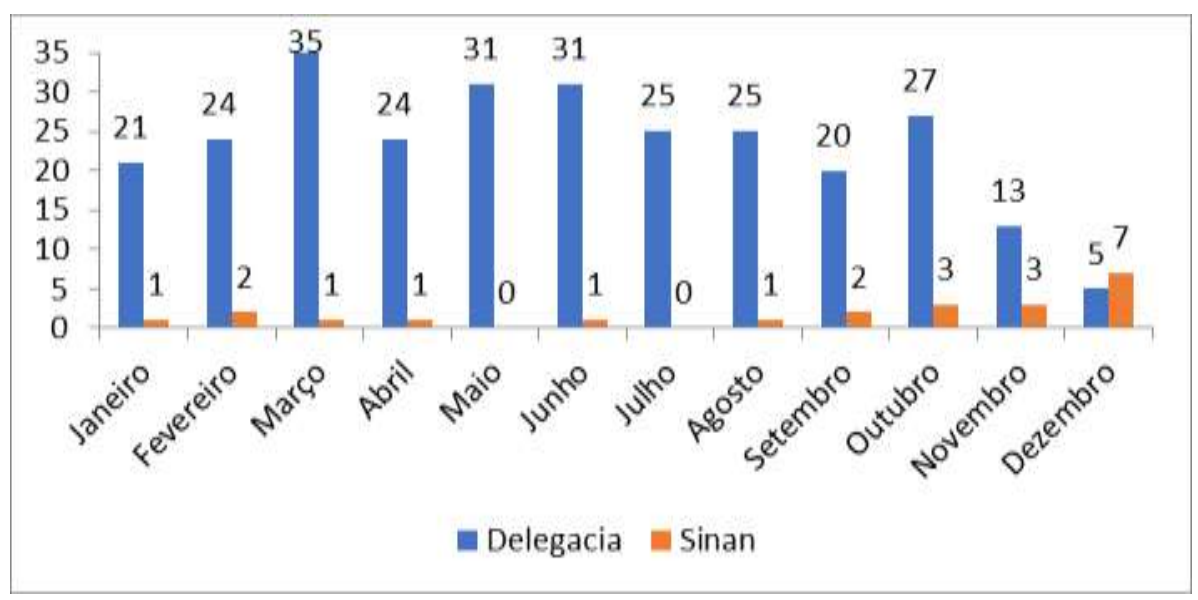

Fonte: DEAM (2018) e SINAN (2018).

Relacionando os dois bancos de dados utilizados identificamos que apenas 7 (sete) mulheres constavam em ambos os bancos. Observa-se que as variáveis raça e escolaridade estão em concordância com predomínio de mulheres pardas e com ensino médio. A variável idade apresentou divergência importante bem como a data de notificação e o estado civil. A variável data de notificação revela uma discordância entre os bancos de dados, apenas um registro tem a data de notificação idêntica entre os bancos, os demais registros apresentam diferença que variam de 18 dias até 9 meses de diferença. As datas do registro da DEAM foram todas anteriores as datas dos SINAN. Em relação ao tipo de violência a divergência verificada decorre dos termos utilizados pelos bancos de dados serem diferentes (Tabela 3).

Tabela 3. Vítimas de violência contra a mulher notificadas no banco de dados da DEAM e do SINAN, no município de Cáceres-MT, 2018.

\begin{tabular}{|c|c|c|c|c|c|c|c|c|}
\hline \multirow[b]{2}{*}{ Variável } & \multirow{2}{*}{$\begin{array}{c}\text { Banco de } \\
\text { dados }\end{array}$} & \multicolumn{7}{|c|}{ Notificações das mulheres encontradas em ambos bancos } \\
\hline & & 1 & 2 & 3 & 4 & 5 & 6 & 7 \\
\hline & DEAM & 25 & - & 29 & 24 & 30 & 46 & 25 \\
\hline Idade & SINAN & 26 & 33 & 30 & 25 & 30 & 46 & 25 \\
\hline Raça & $\begin{array}{l}\text { DEAM } \\
\text { SINAN }\end{array}$ & $\begin{array}{c}- \\
\text { Parda }\end{array}$ & $\begin{array}{c}- \\
\text { Parda }\end{array}$ & $\begin{array}{c}- \\
\text { Parda }\end{array}$ & $\begin{array}{c}- \\
\text { Parda }\end{array}$ & $\begin{array}{l}\text { Parda } \\
\text { Parda }\end{array}$ & $\begin{array}{c}- \\
\text { Parda }\end{array}$ & $\begin{array}{c}- \\
\text { Parda }\end{array}$ \\
\hline Estado civil & $\begin{array}{l}\text { DEAM } \\
\text { SINAN }\end{array}$ & $\begin{array}{l}\text { Viúva } \\
\text { Casada }\end{array}$ & Casada & $\begin{array}{c}\text { Solteira } \\
\text { Casada }\end{array}$ & $\begin{array}{c}\text { Convivente } \\
\text { Solteira }\end{array}$ & $\begin{array}{l}\text { Solteira } \\
\text { Casada }\end{array}$ & $\begin{array}{l}\text { Casada } \\
\text { Casada }\end{array}$ & $\begin{array}{l}\text { Solteira } \\
\text { Solteira }\end{array}$ \\
\hline Escolaridade & $\begin{array}{l}\text { DEAM } \\
\text { SINAN }\end{array}$ & - & $\begin{array}{l}\text { Fundamental } \\
\text { Fundamental }\end{array}$ & Médio & $\begin{array}{l}\text { Médio } \\
\text { Médio }\end{array}$ & $\begin{array}{c}\text { Médio } \\
-\end{array}$ & Médio & $\begin{array}{l}\text { Médio } \\
\text { Médio }\end{array}$ \\
\hline $\begin{array}{l}\text { Data de } \\
\text { notificação }\end{array}$ & $\begin{array}{c}\text { DEAM } \\
\text { SINAN } \\
\text { Diferença }\end{array}$ & $\begin{array}{c}03 / 03 / 2018 \\
25 / 12 / 2018 \\
9 \text { meses }\end{array}$ & $\begin{array}{l}24 / 03 / 2018 \\
24 / 03 / 2018\end{array}$ & $\begin{array}{c}11 / 04 / 2018 \\
07 / 10 / 2018 \\
6 \text { meses }\end{array}$ & $\begin{array}{c}\text { 03/07/2018 } \\
29 / 11 / 2018 \\
4 \text { meses }\end{array}$ & $\begin{array}{c}05 / 07 / 2018 \\
25 / 12 / 2018 \\
5 \text { meses }\end{array}$ & $\begin{array}{c}23 / 09 / 2018 \\
25 / 12 / 2018 \\
3 \text { meses }\end{array}$ & $\begin{array}{c}\text { 11/11/2018 } \\
29 / 11 / 2018 \\
18 \text { dias }\end{array}$ \\
\hline $\begin{array}{l}\text { Tipo de } \\
\text { violência }\end{array}$ & DEAM & $\begin{array}{l}\text { Lesão } \\
\text { corporal }\end{array}$ & $\begin{array}{c}\text { Lesão } \\
\text { corporal } \\
\text { Violência } \\
\text { física + } \\
\text { violência } \\
\text { psicológica }\end{array}$ & $\begin{array}{c}\text { Lesão } \\
\text { corporal } \\
\text { Violência } \\
\text { física + } \\
\text { violência } \\
\text { psicológica }\end{array}$ & $\begin{array}{l}\text { Lesão } \\
\text { corporal }\end{array}$ & $\begin{array}{c}\text { Lesão } \\
\text { corporal } \\
\text { Violência } \\
\text { física + } \\
\text { violência } \\
\text { psicológica }\end{array}$ & $\begin{array}{c}\text { Lesão } \\
\text { corporal } \\
\text { Violência } \\
\text { física + } \\
\text { violência } \\
\text { psicológica }\end{array}$ & $\begin{array}{l}\text { Violência } \\
\text { física }\end{array}$ \\
\hline
\end{tabular}

Fonte: DEAM (2018) e SINAN (2018). 
Após a verificação dos dois bancos ressalta-se que em Cáceres no ano de 2018, apenas 07 mulheres que sofreram violência foram atendidas no sistema policial e no sistema de saúde. Ao todo 296 mulheres sofreram algum tipo de violência física ou sexual, dos quais 274 registros foram realizados apenas na DEAM e 15 registros foram realizados apenas no SINAN.

\section{Discussão}

Este estudo mostra deficiências nas notificações de ocorrências de violência contra mulheres em Cáceres, município de fronteira do Estado de mato Grosso, segundo os serviços de saúde e de segurança pública. Ainda assim é possível identificar o perfil das mulheres que buscaram algum tipo de serviço público para a sua proteção e/ou recuperação.

O primeiro ponto de divergência está relacionado ao número de mulheres que sofreram violência registradas da DEAM e no SINAN, o número da DEAM é 12 vezes maior que os casos registrados do SINAN. Estudo realizado no município de Embu no Estado de São Paulo as mulheres vítimas de violência geralmente procuram ajuda de pessoas próximas ou da própria família. A delegacia foi procurada em 36,8\% dos casos (Burschi; Paula \& Bordin, 2006).

Os serviços de saúde geralmente são utilizados pelas mulheres em situações que as agressões resultaram em lesões importantes, mas geralmente a real causa dessas lesões não é relatada aos profissionais que as atendem (Silva, et al., 2006), o que pode gerar subnotificação, podendo justificar essa diferença entre a DEAM e o SINAN. Pode-se supor também que a população não considera a situação de violência doméstica como uma questão de saúde, mas sim policial (Burschi; Paula \& Bordin, 2006).

Existe a possibilidade da agressão sofrida pela mulher, mesmo que classificada como lesão corporal pela DEAM, não tenha sido grave o suficiente para ter a necessidade do atendimento médico, o que poderia explicar parte dos dados perdidos entre os dois bancos, porém os casos de estupros na DEAM e de violência sexual no SINAN chamam a atenção, pois em nenhum dos casos essas notificações estão nos dois bancos, incluindo os casos de violência sexual em menores.

Os hospitais devem oferecer às vítimas de violência sexual atendimento emergencial, integral e multidisciplinar, visando controle e tratamento dos agravos físicos e psíquicos decorrentes de violência sexual, e encaminhamento, se for o caso, aos serviços de assistência social (Brasil, 2013). O serviço de saúde que atendeu a vítima deve ser responsável por realizar o exame físico e ginecológico, coleta de amostras para diagnóstico de infecções genitais, coleta de material para identificação do provável autor(a) da agressão, e realizar as medicações profiláticas necessárias de acordo com o protocolo de prevenção e tratamento dos agravos resultantes da violência sexual. E não menos importante preencher a Ficha de Notificação e Investigação de Violência Doméstica, Sexual e/ou outras Violências em casos suspeitos ou confirmados (Brasil, 2014).

Após o atendimento médico, a mulher deve ser orientada a procurar a delegacia e realizar o boletim de ocorrência policial, prestar depoimento, ou submeter-se a exame pelos peritos do IML. O boletim de ocorrência policial registra a violência para o conhecimento da autoridade policial, que determina a instauração do inquérito e da investigação. O laudo do Instituto Médico Legal (IML) é documento elaborado para fazer prova criminal (Brasil, 2014).

No caso das mulheres vítimas de violência sua autonomia e sua vontade em compartilhar ou não sua história deve ser respeitada e sua identidade deve ser resguardada. Já no caso de crianças e adolescentes a lei obrigada os profissionais de saúde notificar o Conselho Tutelar casos suspeitos ou confirmados, que fazem o encaminhamento ao sistema policial/judiciário (Brasil, 2014), mesmo com essa obrigatoriedade nenhum caso de estupro em menores notificado na DEAM foi registrado pelo SINAN.

Todas as pessoas têm o dever de notificar as autoridades quando ocorre algum caso de violência, mas a Lei 10.778/2003 define a obrigação dos serviços de saúde públicos ou privados de notificar os casos suspeitos ou confirmados de violência contra a mulher, pois a notificação pode gerar políticas de prevenção e controle desses casos (Oliveira, et al., 2018). 
Um estudo realizado em 10 municípios brasileiros através de questionários, verificou o alto número de profissionais que não adotaram nenhuma atitude para a condução dos casos suspeitos ou confirmados de violência (Souza, et al., 2018). As mulheres afirmam o desejo de serem questionadas pelos profissionais de saúde sobre agressões e maus tratos, o que pouco ocorre no âmbito dos serviços de saúde (Silva, et al., 2012).

Os profissionais de saúde referem várias justificativas para a não notificação dos casos de violência contra a mulher, dentre elas: desconhecimento da ficha de notificação, medo de retaliações, dificuldade ou constrangimento para preencher a ficha, sobrecarga no cotidiano do serviço e dificuldade em lidar com os casos (Kind, et al., 2013). Um trabalho realizado em Londres e Bristol na Inglaterra, com profissionais da saúde, constatou a falta de preparo desses profissionais para atender às necessidades de mulheres em situação de violência (Ramsay, et al., 2012). Neste estudo identificamos uma grande diferença nas datas de notificação dos casos encontrados em ambos os bancos de dados e a maioria das notificações do SINAN ocorram um tempo depois da notificação na DEAM. É possível levantar hipóteses para esse fato, ou as agressões ocorreram em momentos diferentes e essas mulheres em cada momento procuram um serviço diferente, ou que as notificações no serviço de saúde foram realizadas um tempo depois da agressão, ou ainda que ocorrem erros de digitação referente a data de notificação.

Outra questão é como as violências são classificadas pelo sistema judiciário e pelo sistema de saúde. Nos registros dos boletins de ocorrências realizados na DEAM as violências são classificadas de acordo com o Código Penal Brasileiro em injúria, calúnia, lesão corporal e estupro (Brasil, 2002), segundo o artigo 129 lesão corporal é o ato de ofender a integridade corporal ou a saúde de outrem, e o artigo 213 caracteriza estupro o ato de constranger mulher a conjunção carnal, mediante violência ou grave ameaça (Brasil, 2002).

Para o preenchimento da ficha do SINAN as definições são de acordo com a natureza da violência e são classificadas em violência física, violência psicológica/moral e violência sexual (Brasil, 2016). Percebe-se que apesar dos diferentes termos utilizados pelo SINAN e pela DEAM há uma semelhança nas suas descrições, não causando dificuldade no momento da notificação. Para um melhor levantamento epidemiológico do tipo de violência que a mulher é vítima, parece ser necessária uma padronização dos termos utilizados nos serviços de segurança com o de saúde.

Mas apesar de ser possível comparar os bancos de dados pelo tipo de violência sofrida, os profissionais de saúde no momento do preenchimento do respectivo campo podem apresentar dificuldades no preenchimento da ficha e por isso muitas vezes optem pelo item "ignorado" ou acabam deixando em branco (Delziovo, et al., 2018).

Estudo realizado em Recife-PE para verificar a qualidade dos dados do SINAN mostrou que a deficiência na qualidade dos dados pode estar relacionada com a falta de compromisso dos profissionais com a obrigatoriedade da notificação (Abath, et al., 2014).

Alguns erros na ficha de notificação também podem ser ocasionados por erro de digitação e outras pela própria estrutura da ficha. Por exemplo, no campo tipo de violência tem a opção de marcar: "sim", "não" e ignorado para cada tipo de violência, o que induz o profissional a registrar mais de um tipo de violência, embora a recomendação seja registrar apenas o tipo principal ${ }^{36}$. Esse fato pode ser observado em nosso estudo onde as fichas do SINAN apresentaram associação de violência física com as demais (psicológica, sexual) e associação de violência sexual e psicológica.

O perfil das mulheres que sofrem violência é bem definido independente do banco de dados analisado, caracteriza-se por mulheres pardas, com idade entre 21 a 30 anos, casadas ou conviventes, com ensino fundamental e do lar.

Ao analisar a prevalência de acordo com a idade das mulheres no presente estudo a faixa etária mais acometida está entre 21 e 30 anos semelhante ao que foi encontrado em um estudo realizado através das notificações no Rio Grande do Sul cuja faixa etária demonstraram maior notificação entre vítimas de 19 a 29 anos (Lawrenz, et al., 2018). Resultados semelhantes também foram encontrados em um estudo realizado com os registros de uma Delegacia Civil de Montes Claros em que $80 \%$ dos casos ocorreram na faixa etária adulta (Leite, et al., 2014). 
As menores taxas de violência ficaram nos extremos da vida entre as menores de 10 anos $(4,6 \%)$ e as maiores de 50 anos $(5,3 \%)$. Estando em concordância com um estudo do Rio Grande do Sul onde as menores taxas de violência ficaram entre 50 a 59 anos (Lawrenz, et al., 2018).

Uma das explicações para o fato da violência ocorrer mais na faixa etária adulta pode estar relacionada ao fato de que essas mulheres se encontram em um período de maior atividade sexual e reprodutiva (Leite, et al., 2014).

Neste estudo podemos verificar que existem alguns casos de violência física e sexual contra crianças menores de 10 anos e o que chama atenção é que esses dados não foram encontrados no banco de dados do SINAN. A notificação de crianças e adolescentes em situação de violência é regulamentada pela Lei no 8.069/1990 - Estatuto da Criança e do Adolescente (ECA), que foi regulamentado pelo MS através da Portaria MS n ${ }^{\circ}$ 1.968/2001 e uma cópia da ficha de notificação deve ser encaminhada ao Conselho Tutelar da Criança e do Adolescente conforme preconiza o ECA (Brasil, 2014).

Diversos estudos relacionam a baixa escolaridade com o maior risco de sofrer violência. Um estudo realizado em serviços de urgência e emergência de capitais brasileiras mostrou que entre os fatores de risco para violência doméstica era ter menos de 8 anos de estudo e não exercer atividade remunerada (Garcia, et al., 2016). O que está em concordância com nosso estudo, onde tanto as vítimas como os agressores apresentam apenas o ensino fundamental. Uma revisão bibliográfica realizada na Etiópia mostra que mulheres com escolaridade maior tem menor probabilidade de sofrer violência pelos seus parceiros (Semahegn \& Mengistie, 2015).

Quanto à ocupação, estudos apontam que mulheres que desenvolvem atividade profissional remunerada buscam estratégias mais eficazes para enfrentar a situação de violência. Um estudo realizado com as fichas do setor de psicologia de uma delegacia na região metropolitana de Porto Alegre evidenciou que 43,3\% têm empregos em comércio e indústria, 32,5\% são donas de casa ou não têm profissão (Gadoni-Costa; Zucatti \& Dell'Aglio, 2011). O que é semelhante com nosso estudo onde a maior parte das vítimas são do lar seguido por trabalhadores de serviço do comércio, em lojas e mercados.

Mulheres casadas e conviventes apresentaram maior prevalência entre as mulheres vítimas de violência, o que está em discordância com um estudo realizado em Vitória no Espírito Santos que demonstrou maior prevalência de violência nas mulheres divorciadas ou separadas (Leite, et al., 2017).

Houve uma maior prevalência de violência em mulheres pardas, não havendo muita associação da violência com a cor da pele o que foi identificado também em outros estudos (Vieira; Perdona \& Santos, 2011; Leite, et al., 2017).

Mulheres com classes mais favorecidas e com mais anos de escolaridade dispõem de recursos que possibilitam encontrar ajuda de outra forma, o que não significa que mulheres com melhor nível socioeconômico não enfrentam situações de violência, mas que existe uma sub-representação das denúncias (Gadoni-Costa; Zucatti \& Dell’Aglio, 2011).

Verificou-se neste estudo, entre as formas de violência estudadas, a prevalência maior foi de lesão corporal segundo a DEAM e segundo o SINAN o tipo de violência mais sofrido pelas mulheres foi violência física e a associação de violência física e psicológica, e física e sexual. O que é consistente com os resultados de outro estudo realizado na região nordeste do Brasil com os dados de uma DEAM que apresentaram a violência física como sendo a mais prevalente (Bernadino, et al., 2016). Já no município de Vitória- ES a violência psicológica foi a mais frequente $(25,3 \%)$, seguida pela violência física $(9,9 \%)$ e violência sexual teve a menor prevalência (5,7\%) (Leite, et al., 2017).

Um estudo realizado na Turquia identificou a violência física como a lesão mais prevalente, sendo cometida pelos seus parceiros íntimos em primeiro lugar e em segundo lugar pelos seus pais (homens) (Sem \& Bolsoy, 2017).

No município de São Paulo uma investigação com usuárias de uma unidade básica de saúde com idade entre 15 e 49 anos, encontrou $44,4 \%$ das usuárias relatando ocorrência de episódio de violência física e $11,5 \%$ de violência sexual (Marinheiro; Vieira \& Souza, 2006). 
Neste estudo podemos ver todos os agressores foram homens raça/cor da pele parda, com idade entre 31 a 40 anos, casados/conviventes, com ensino fundamental e trabalhadores de serviços, vendedores do comércio em lojas e mercados. Um estudo realizado na região Nordeste do Brasil evidenciou que quase todos os agressores eram homens (93\%), confirmando que as mulheres são mais agredidas por homens, os quais na maioria das vezes possuem alguma relação íntima com a vítima (Bernardino, et al., 2016).

Uma revisão da literatura mostrou que a maioria dos agressores eram homens com idade entre 30 e 49 anos (Cavalcanti, et al., 2020), o que concorda com nosso estudo onde a maior prevalência foi entre 31 e 40 anos. Essa revisão ainda mostrou que a maioria dos agressores eram de cor branca e com grau de escolaridade completo (Cavalcanti, et al., 2020), o que difere do presente estudo em que a maioria são da cor/raça parda e o grau de escolaridade ensino fundamental.

Os principais agressores são os parceiros íntimos, tanto atuais quanto ex-parceiros ${ }^{41}$. Essa pesquisa não pode evidenciar o grau de parentesco com a vítima devido os dados contidos no boletim de ocorrência da DEAM e nas fichas de notificação do SINAN, o qual foram os bancos de dados utilizados, não incluíram essa informação.

Quanto as limitações do estudo podemos levar em consideração o desenho metodológico ser do tipo descritivo e focalizar apenas na identificação do problema, mas não contempla exatamente como o problema ocorreu, além de que a amostra é muito variável e dessa forma os resultados devem ser interpretados com cautela, já que eles ressaltam apenas os casos em que as vítimas decidiram procurar o serviço. O estudo optou-se por avaliar apenas dois tipos de violência, mas se tratando de um estudo que tem por objetivo comparar a confiabilidade do banco de dados da DEAM e do SINAN preferiu utilizar apenas esses dois tipos de violência pois seria os tipos que poderiam estar presentes nos dois bancos de dados. Todos os casos foram tratados como casos novos, por ser um estudo transversal não foi verificado se o caso for recorrente ou realmente caso novo.

Outra dificuldade encontrada está relacionada ao grande número de dados ignorados nos bancos de dados analisados e também o pequeno número de casos notificados no SINAN. Como muitas variáveis foram deixadas em branco, isso pode interferir no perfil das mulheres vítimas de violência. Como muitos dados encontrados no banco de dados da DEAM não estavam presentes no SINAN fica difícil esclarecer se realmente ocorreram casos de subnotificação ou se essas mulheres não procuraram o serviço de saúde.

Espera-se que essas informações sejam utilizadas para melhorar o conhecimento a respeito da violência contra a mulher, confirmando a importância da notificação e a necessidade de ações articuladas e intersetoriais sendo fundamental a comunicação entre o serviço de saúde e os setores judicial e policial, bem como ocorre com as violências contra a criança e contra o idoso. Desta forma, a articulação desses setores é indispensável para a resolução da violência contra mulher. Os serviços de saúde de acordo com a Lei 13.931 que entrou em vigor em 10 de maio de 2020 (Fórum Brasileiro de Segurança Pública, 2019) devem por obrigação comunicar os casos em que houver indícios ou confirmação de violência contra a mulher às autoridades policiais no prazo de 24 (vinte e quatro) horas.

Para reduzir ainda mais os casos subnotificados a também a necessidade do setor policial comunicar o serviço de saúde e estimular essas mulheres a procurar atendimento em unidades básicas de saúde ou hospitais para que dessa forma os casos sejam notificados em ambos os setores e a violência contra a mulher não fique invisível para as ações de políticas públicas.

Entre as limitações é provável que o perfil de mulheres que sofreram violência esteja enviesado em razão do sub registros. Possivelmente mulheres de maior renda e/ou mais escolarizadas não busquem ajuda em razão do estigma ligados às agressões.

Para reduzir a invisibilidade da violência contra a mulher no serviço de saúde é necessário que sejam adotadas estratégias para estimular e melhorar o preenchimento da ficha de notificação. São algumas dessas estratégias: capacitação dos 
profissionais de saúde em relação a legislação brasileira (código penal) bem como a lei referente a notificação dos casos de violência contra a mulher e para que ocorra o adequado preenchimento da ficha; treinamento e sensibilização desses profissionais para que eles reconheçam mulheres em situações de violência e a importância desses registros; monitoramento e cobrança por parte da equipe de vigilância epidemiológica quando identificar o pequeno número de casos notificados no mês e da qualidade do banco de dados (Abath, et al., 2014). Outros estudos parecem serem necessários para avaliar as causas das discrepâncias entre os dados, ou ainda, analisar a qualidade na coleta dos dados para os sistemas judiciário e de saúde.

\section{Conclusão}

Conclui-se que a violência física foi o tipo de violência mais frequente seguida da violência sexual. Este estudo aponta discordâncias entre os registros de violência contra mulher, sugerindo importante subnoficação do agravo, além da falta de integração das áreas de segurança pública e de saúde.

\section{Referências}

Abath, M. B., Lima, M. L. L. T., Lima, P. S., Silva, M. C. M. \& Lima, M. L. C. (2014). Avaliação da completitude, da consistência e da duplicidade de registros de violências do Sinan em Recife, Pernambuco, 2009-2012. Epidemiol. Serv. Saúde. 23(1), 131-142.

Bernardino, Í. M., Barbosa, K. G. N., Nóbrega, L. M., Cavalcante, G. M. S., Ferreira, E. F. \& d'Avila, S. (2016). Violência contra mulheres em diferentes estágios do ciclo de vida no Brasil: um estudo exploratório. Rev Bras Epidemiol, 19(4):740-752.

Brasil. (2002). Decreto-Lei no 2.848, de 7 de dezembro de 1940. Institui o Código Penal Brasileiro. Diário Oficial da União: seção 1, Brasília, DF, 139(8):174.

Brasil. (2002). Ministério da Saúde. Violência intrafamiliar: orientações para a prática em serviço. Brasília (DF): Cadernos de Atenção Básica, 8.

Brasil. (2006) Lei ${ }^{\circ}$ 11.340, de 07 de agosto de 2006. Lei Maria da Penha: cria mecanismos para coibir a violência doméstica e familiar contra a mulher. Diário Oficial da União. Brasília.

Brasil. (2013). Lei No 12.845, de $1^{\circ}$ de agosto de 2013. Diário Oficial da União. Brasília.

Brasil. (2014). Ministério da Saúde. Secretaria de Atenção à saúde. Departamento de Ações Programáticas Estratégicas. Prevenção e tratamento dos agravos resultantes da violência sexual contra mulheres e adolescentes: norma técnica. 3. Ed. Brasília: Ministério da Saúde.

Brasil. (2016). Secretaria de Vigilância em Saúde. Departamento de Vigilância de Doenças e Agravos Não Transmissíveis e Promoção da Saúde. Viva: instrutivo de notificação de violência interpessoal e autoprovocada. 2. ed. Brasília: Ministério da Saúde.

Brasil. (2019). Lei № 13.931, de 10 de dezembro de 2019. Diário Oficial da União. Brasília.

Burschi, A., Paula, C. S. \& Bordin, I. A. S. (2006). Prevalência e Procura de ajuda na violência conjugal física ao longo da vida. Ver. Saúde Pública, 40(2), 256-64.

Cavalcanti, G. M. B., Amorim, A. V. B., Queiroz, G. S., Cruz, N. M.; Costa, R. L. \& Bezerra, K. F. O. (2020). A violência contra a Mulher no Sistema Único de Saúde. Rev Fun Care Online, 146-154.

Delziovo, C. R., Bolsoni, C. C., Lindner, S. R. \& Coelho, E. B. S. (2018). Qualidade dos registros de violência sexual contra a mulher no Sistema de Informação de Agravos de Notificação (Sinan) em Santa Catarina, 2008-2013. Epidemiol. Serv. Saude, Brasília, 27(1), e20171493.

Fórum Brasileiro de Segurança Pública. (2019). Visível e Invisível: A vitimização de mulheres no Brasil. $2^{\mathrm{a}}$ ed.

Gadoni-Costa, L. M., Zucatti, A. P. N. \& Deell'Aglio, D. D. (2011). Violência contra a mulher: levantamento dos casos atendidos no setor de psicologia de uma delegacia para a mulher. Estudos de Psicologia I, 28(2), 219-227

Garcia, L. P., Duarte, E. C., Freitas, L. R. S. \& Silva, G. D. M. (2016). Violência doméstica e familiar contra a mulher: estudo de casos e controles com vítimas atendidas em serviços de urgência e emergência. Cad. Saúde Pública, Rio de Janeiro, 32(4), e00011415.

Gil, A. C. (2017). Como elaborar projetos de pesquisa. (6a ed.), Atlas.

Girianelli, V. R., Ferreira, A. P., Vianna, M. B., Teles, N., Erthal, R. M. C. \& Oliveira, M. H. B. (2018). Qualidade das notificações de violências interpessoal e autoprovocada no Estado do Rio de Janeiro, Brasil, 2009-2016. Cad. Saúde Colet., 26(3),318-326.

Instituto Brasileiro de Geografia e Estatística (2018). Cidades: Cáceres. https://cidades.ibge.gov.br/brasil/mt/caceres/panorama>.

Kind, L., Orsini, M. L. P., Nepomuceno, V., Gonçalvez, L., Souza, G. A. \& Ferreira, M. F. F. (2013). Subnotificação e (in)visibilidade da violência contra mulheres na atenção primária à saúde. Cad. Saúde Pública, Rio de Janeiro, 29(9), 1805-1815. 
Research, Society and Development, v. 10, n. 3, e0910312941, 2021

(CC BY 4.0) | ISSN 2525-3409 | DOI: http://dx.doi.org/10.33448/rsd-v10i3.12941

Lawrenz, P., Macedo, D. M., Hohendorff, J. V., Freitas, C. P. P., Foschiera, L. N. \& Habigzang, L. F. (2018). Violência contra Mulher: Notificações dos Profissionais da Saúde no Rio Grande do sul. Psic.: Teor. e Pesq., Brasília, 34: e34428.

Leite, F. M. C., Amorim, M. H. C., Wehrmeister, F. C. \& Gigante, D. P. (2017). Violence against women, Espírito Santo, Brazil. Rev Saúde Pública, 51:33.

Leite, M. T. S., Figueiredo, M. F. S., Dias, O. V., Vieira, M. A., Souza e Souza, L. P. \& Mendes, D. C. (2014). Reports of violence against women in different life cycles. Rev Lat Am Enfermagem. 22(1):85-92.

Marinheiro, A. L. V., Vieira, E. M., \& Souza, L. (2006). Prevalência da violência contra a mulher usuária de serviço de saúde. Rev Saúde Pública, 40(4):60410 .

Meneghel, S. N., Mueller, B., Collaziol, M. E., \& Quadros, M. M. (2013). Repercussões da Lei Maria da Penha no enfrentamento da violência de gênero. Ciênc Saúde Coletiva, 18(3):691-700.

Oliveira, B. G., Freire, I. V., Assis, C. S., Sena, E. L. S., Boery, R. N. S. O. \& Yarid, S. D. (2018). Responsabilidade dos profissionais de saúde na notificação dos casos de violência. Rev. bioét. (Impr.), 26 (3): 403-11.

Pereira, A. S. et al. (2018). Metodologia da pesquisa científica. UFSM.

Ramsay, J., Rutterford, C., Gregory, A., Dunne, D., Eldridge, S., Sharp, D. \& Feder, G. (2012). Domestic violence: knowledge, attitudes, and clinical practice of selected UK primary healthcare clinicians. British Journal of General Practice.

Saliba, O., Garbin, C. A. S., Garbin, A. J. I. \& Dossi, A. P. (2007). Responsabilidade do profi ssional de saúde sobre a notificação de casos de violência doméstica. Rev Saúde Pública, 41(3):472-7.

Semahegn, A. \& Mengistie, B. (2015). Domestic violence against women and associated factors in Ethiopia; systematic review. Semahegn and Mengistie Reproductive Health. Dezembro, 12(78).

Sen, S. \& Bolsoy, N. (2017). Violence against women: prevalence and risk factors in Turkish sample. BMC Womens Health, 17(1).

Silva, L. E. L. \& Oliveira, M. L. C. (2015). Violência contra a mulher: revisão sistemática da produção científica nacional no período de 2009 a 2013 . Ciênc Saúde Coletiva. Novembro, 20(11):3523-3532.

Silva, R. A., Araújo, T. V. B., Valongueiro, S. \& Ludermir, A. B. (2012). Enfrentamento da violência infligida pelo parceiro íntimo por mulheres em área urbana da região Nordeste do Brasil. Rev Saúde Pública, 46(6),1014-22.

Souza, E.G., Tavares, R., Lopes, J. G., Magalhães, M. A. N. \& Melo, E. M. (2018). Atitudes e opiniões de profissionais envolvidos na atenção à mulher em situação de violência em 10 municípios brasileiros. Saúde Debate, 42(4):13-29.

Vieira, E. M., Perdona, G. S. C. \& Santos, M. A. (2011). Fatores associados à violência física por parceiro íntimo em usuárias de serviços de saúde. Rev Saúde Pública, 45(4):730-7.

Vieira, L. J. E. S., Ferreira, R. C., Moreira, G. A. R., Gondim, A. P. S., Araujo, M. A. L. \& Silva, R. M. S. (2013). Fatores associados à sobreposição de tipos de violência contra a mulher notificada em serviços sentinel. Rev. Latino-Am. Enfermagem jul-ago, 21(4).

Waiselfisz, J. J. (2015). Mapa da violência 2015: homicídio de mulheres no Brasil. (DF).

World Health Organization. (1996.) Violence: a public health priority: WHO Consultation on Violence and Health. Geneva. 